\title{
Impactos psicossociais do isolamento social por COVID-19 em crianças, adolescentes e jovens: scoping review
}

Psychosocial impacts of social isolation due to COVID-19 in children, adolescents and young people: A scoping review

Repercusiones psicosociales del aislamiento social debido al COVID-19 en niños, adolescentes y jóvenes: scoping review

\section{Letícia Camilo Santos ${ }^{I}$, Tayná July Silva Pinheiro"I, Thayane Ingrid Xavier de Andrade ${ }^{\mathrm{III}}$ Paulo Henrique Alves Sousa ${ }^{\mathrm{IV}}$, Patrícia Pinto Bragav , Márcia Christina Caetano Romano ${ }^{\mathrm{VI}}$}

\begin{abstract}
Resumo: Objetivo: mapear os impactos psicossociais causados pelo isolamento social, durante a pandemia da COVID19, em crianças, adolescentes e jovens. Método: revisão de escopo realizada de acordo com o método proposto por JBI e adotando as recomendações do guia internacional Preferred Reporting Items for Systematic reviews and Meta-Analyses extension for Scoping Reviews. Protocolo de revisão registrado no Open Science Framework. Resultados: compuseram esta revisão 20 artigos. A depressão $(70 \%$; $n=14)$ e a ansiedade $(60 \% ; n=16)$ foram os sintomas mais frequentes entre as investigações estudadas. Identificou-se um aumento do tempo de tela e utilização de internet. Conclusão: o isolamento social em função da COVID-19 tem impactado social e psicologicamente na vida de crianças e adolescentes. O adoecimento mental deste público e as repercussões no desenvolvimento infanto-juvenil não podem ser desconsideradas. Descritores: Criança; Adolescente; Isolamento Social; Infecções por Coronavírus; Impacto Psicossocial
\end{abstract}

\footnotetext{
Abstract: Objective: to map the psychosocial impacts caused by social isolation during the COVID-19 pandemic in children, adolescents and young people. Method: a scoping review conducted according to the method proposed by the JBI and adopting the recommendations set forth in the Preferred Reporting Items for Systematic reviews and MetaAnalyses extension for Scoping Reviews international guide. The review protocol was registered in the Open Science Framework. Results: a total of 20 articles comprised this review. Depression (70\%; n=14) and anxiety (60\%; n=16) were the

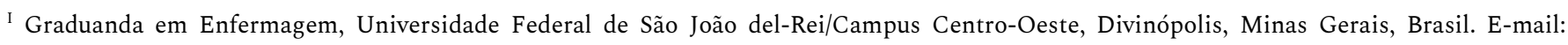
leticiacamilosantos2012@gmail.com, Orcid: https://orcid.org/0000-0002-9113-7453

II Graduanda em Enfermagem, Universidade Federal de São João del-Rei/Campus Centro-Oeste, Divinópolis, Minas Gerais, Brasil. E-mail: taynajuly03@gmail.com, Orcid: https://orcid.org/0000-0002-0673-1395

III Graduanda em Enfermagem, Universidade Federal de São João del-Rei/Campus Centro-Oeste, Divinópolis, Minas Gerais, Brasil. E-mail: thatha.red@gmail.com, Orcid: https://orcid.org/0000-0002-6561-7509

IV Nutricionista, Mestrando. Universidade Federal de São João del-Rei/Campus Centro-Oeste, Divinópolis, Minas Gerais, Brasil. E-mail: spaulohenrique@hotmail.com, Orcid: https://orcid.org/0000-0003-3077-3947

V Enfermeira, Pós-Doutora. Professora. Universidade Federal de São João del-Rei/Campus Centro-Oeste, Divinópolis, Minas Gerais, Brasil. Email: patriciabragaufsj@gmail.com, Orcid: https://orcid.org/0000-0002-1756-9186

VI Enfermeira, Doutora. Professora. Universidade Federal de São João del-Rei/Campus Centro-Oeste, Divinópolis, Minas Gerais, Brasil. E-mail: marciachristinacs@gmail.com, Orcid: https://orcid.org/0000-0002-1819-4689
} 
Impactos psicossociais do isolamento social por COVID-19 em crianças, adolescentes... | 2

most frequent symptoms among the research studies evaluated. An increase in screen time and in Internet use was identified. Conclusion: social isolation due to COVID-19 has exerted social and psychological effects on children's and adolescents' lives. In this population, mental ailments and their repercussion in child-youth development cannot be disregarded.

Descriptors: Child; Adolescent; Social Isolation; Coronavirus Infections; Psychosocial Impact

Resumen: Objetivo: mapear las repercusiones psicosociales causadas por el aislamiento social durante la pandemia de COVID-19 en niños, adolescentes y jóvenes. Método: scoping review realizada de acuerdo con el método propuesto por el JBI y en la que se adoptaron las recomendaciones de la guía internacional Preferred Reporting Items for Systematic reviews and Meta-Analyses extension for Scoping Reviews. El protocolo de revisión se registró en Open Science Framework. Resultados: esta revisión estuvo compuesta por 20 artículos. Depresión (70\%; n=14) y ansiedad (60\%; n=16) fueron los síntomas más frecuentes en las investigaciones del estudio. Se identificó un aumento en el tiempo que se pasa frente a las pantallas de diversos dispositivos y en el uso de Internet. Conclusión: el aislamiento social debido al COVID19 ha afectado social y psicológicamente la vida de niños y adolescentes. Las enfermedades mentales en esta población son una realidad, y las repercusiones en el desarrollo infanto-juvenil no pueden ignorarse.

Descriptores: Niño; Adolescente; Aislamiento Social; Infecciones por Coronavirus; Impacto Psicosocial

\section{Introdução}

O mundo enfrenta uma grave crise devido à infecção pelo novo coronavírus, ocasionando a mais recente doença infecciosa denominada COVID-19. Este agravo tem afetado populações de modo global. ${ }^{1}$ Provocada pelo SARS-CoV-2, pode caracterizar-se por infecções brandas, moderadas e graves. Grande parte dos infectados apresentam sintomas brandos ou nenhum, mas os casos mais graves demandam atendimento hospitalar, podendo requerer suporte ventilatório. ${ }^{2}$

Segundo dados da Organização Pan-Americana da Saúde (OPAS) em 12 de fevereiro de 2021, no mundo, havia cerca de 107.423 .526 casos confirmados e 2.360 .280 vidas perdidas. ${ }^{3}$ Na população infantil e juvenil no Brasil, até dezembro/2020, 13.276 crianças e adolescentes na faixa etária de 0 a 19 anos foram hospitalizados devido à COVID-19, sendo que 1.118 óbitos foram confirmados. ${ }^{4}$

O coronavírus parece afetar em menor proporção a saúde de crianças e adolescentes, acarretando sintomas menos graves da doença nessa faixa etária em comparação com a população adulta. ${ }^{5}$ No que tange à infectabilidade, acredita-se que na infância há a mesma probabilidade de se infectar com o vírus que os adultos. ${ }^{6}$ Foi evidenciado que indivíduos com idade menor que 18 anos infectados pelo novo coronavírus podem desenvolver uma condição inflamatória multissistêmica, 
com algumas características semelhantes às da doença de Kawasaki e da síndrome do choque tóxico. ${ }^{7}$ Estudos descreveram uma apresentação de doença aguda, acompanhada de uma síndrome hiperinflamatória, levando à falência múltipla de órgãos e choque. ${ }^{7-9}$

Considerando estas características da doença no público infantil e jovem é preciso destacar que a pandemia da COVID-19 impeliu a sociedade a novos modos de vida na tentativa de impedir a disseminação da doença. O isolamento social, restrições de contato, paralizações e fechamento das escolas impõem mudanças no cotidiano das pessoas em todo o mundo, e restringe a socialização na infância e juventude. Essas medidas de mitigação têm o potencial de ameaçar a saúde mental.1,10-12

O processo de socialização contribui para a evolução de aspectos éticos, morais e culturais para se viver em sociedade e contribui para a formulação da identidade e autonomia. E a maneira privilegiada de detectar este desenvolvimento se dá a partir dos encontros com pessoas da família, da escola e comunidade em geral. ${ }^{13}$

Com a ausência da rotina escolar e distanciamento físico, tem-se observado o aumento da utilização de tecnologias, como celulares, computadores e jogos eletrônicos por crianças e adolescentes. De fato, a exacerbação do uso da televisão e mídias interativas portáteis, como smartphone e tablet, tem sido verificada, inclusive em crianças menores de dois anos de idade. ${ }^{14}$

Durante a pandemia, são observadas maiores demandas de saúde voltadas para problemas psicológicos, como ansiedade, medo e estresse, além de mudanças nos aspectos sociais, como a substituição da interação física pela digital. Certamente, essas alterações psicossociais têm o potencial de influenciar o desenvolvimento. ${ }^{15}$

A pandemia da COVID-19 é uma situação atípica no mundo, talvez por isso, ainda são escassos estudos que abordem, analisem e apontem os impactos do isolamento social nos aspectos psicossociais de crianças e adolescentes. ${ }^{16}$ Considerando o exposto este estudo teve como objetivo mapear os impactos psicossociais causados pelo isolamento social, durante a pandemia da COVID19, em crianças, adolescentes e jovens. 
Impactos psicossociais do isolamento social por COVID-19 em crianças, adolescentes... $\mid 4$

\section{Método}

Trata-se de uma revisão de escopo orientada pelo método JBI e que adotou o Preferred Reporting Items for Systematic reviews and Meta-Analyses extension for Scoping Reviews (PRISMA-ScR). Previamente à coleta dos dados o protocolo de revisão foi registrado no Open Science Framework. ${ }^{17-19}$ A revisão ocorreu em cinco etapas: identificação de pesquisa; identificação de estudos relevantes; seleção dos estudos; análise dos dados; e agrupamento, síntese e apresentação dos dados.

Foi utilizada para formulação da pergunta de pesquisa a estratégia mnemônica participantes, conceito e contexto (PCC), sendo P - Crianças e Adolescentes, C - Impactos psicossociais de isolamento social e C - Pandemia Mundial pela Covid-19. ${ }^{18}$ Deste modo, a pergunta de pesquisa definida foi: quais são os impactos psicossociais do isolamento social em crianças, adolescentes e jovens decorrentes da pandemia de COVID-19? Considerou-se criança o indivíduo com até 12 anos de idade incompletos e adolescente aquele com 12 a 18 anos. ${ }^{20}$

Os critérios de inclusão foram pesquisas com textos completos publicados na íntegra em inglês, português ou espanhol com a população de estudo, crianças e adolescentes. Foram incluídas também investigações com participantes na faixa juvenil, ou seja, até 24 anos, desde que em sua amostra tivessem indivíduos de zero a 18 anos de idade. Estipulou-se como limite temporal publicações a partir de dezembro de 2019, quando foi divulgado o primeiro caso da Covid-19 no mundo. ${ }^{3}$ Foram excluídos na seleção editoriais e cartas.

A busca foi realizada no mês de setembro de 2020, por três pesquisadoras independentes, na Biblioteca Virtual em Saúde (BVS) e nas seguintes bases de dados: U.S. National Library of Medicine (PubMed), Web of Science, Scopus, Online Medical Literature Search and Analysis System (Medline), Cumulative Index to Nursing and Allied Health Literature (CINAHL). Todas as bases de dados citadas foram acessadas por meio do Portal de Periódicos da Coordenação de 
5 | Santos LC, Pinheiro TJS, Andrade TIX, Sousa PHA, Braga PP, Romano MCC

Aperfeiçoamento de Pessoal do Nível Superior (CAPES), com seleção de acesso proveniente de instituição de ensino superior. Também foi realizada a busca na literatura cinzenta das páginas oficiais do Ministério da Saúde, Sociedade Brasileira Pediatria, American Academy of Pediatrics, OPAS e Organização Mundial de Saúde.

Os termos de busca foram obtidos dos MeSH, sendo as seguintes estratégias adotadas: (“child” [Mesh] OR “children” [Mesh] AND “social isolation” [Mesh] AND “teenager” [Mesh] OR “teenagers” [Mesh] AND “psychosocial deprivation” [Mesh] OR “psychosocial deprivations” [Mesh] AND “covid-19” [Mesh]. A estratégia de busca foi adaptada conforme as especificidades de cada base e manteve-se a combinação similar dos descritores.

Os dados obtidos na busca foram importados para o aplicativo gratuito Rayyan $^{\circledR}$, disponível na web (https://rayyan.qcri.org/), para a organização e triagem dos artigos. Em seguida, dois pesquisadores, realizaram a leitura de títulos e resumos de todas as publicações disponíveis, selecionando aqueles potencialmente elegíveis para o estudo. As dúvidas de seleção foram decididas em plenária com a participação de um terceiro pesquisador. Realizados os consensos da seleção das publicações, elas foram lidas na íntegra.

Posteriormente foi realizada a separação e extração dos dados dos artigos selecionados, por meio de um formulário estruturado de coleta de dados, elaborado pelos autores, em que se coletaram as seguintes informações: base de dado pesquisada, título, ano, autores, país, tipo de publicação, desenho do estudo, duração do seguimento, local, descrição da amostra, exposição observada, instrumento utilizado para coleta dos dados, resultados, recomendações.

Seguiu-se então com a análise narrativa dos dados com posterior discussão dos resultados com bibliografia pertinente. Ressalta-se que não foi necessária a apreciação ética, por se tratar de um estudo com dados de domínio público. 
Impactos psicossociais do isolamento social por COVID-19 em crianças, adolescentes... | 6

\section{Resultados}

A busca inicial nas bases de dados gerou um total de 1.794 publicações elegíveis. Foram selecionadas 20 publicações ${ }^{21-40}$ que compõem a amostra deste estudo (Figura 1). Não foram identificadas publicações de literatura cinzenta que respondessem à pergunta do estudo.

Figura 1 - Fluxo referente ao processo de seleção dos estudos da Scoping Review, adaptado do PRISMA-ScR. Divinópolis, Minas Gerais, Brasil, 2021
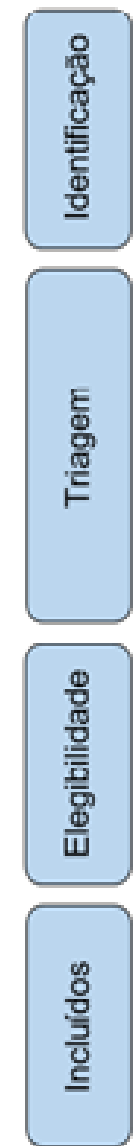

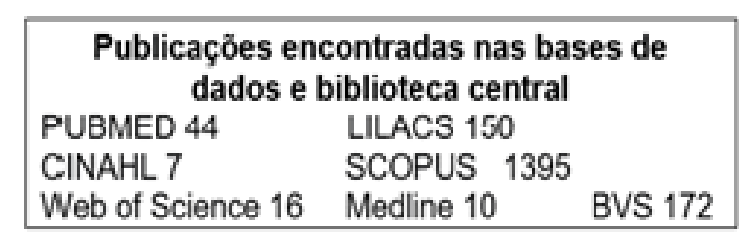

\begin{tabular}{|lll|}
\hline \multicolumn{3}{|c|}{ Exclusăo por duplicaçăo } \\
PUBMED 17 & LILACS 40 \\
CINAHL_5 & SCOPUS 601 & \\
Web of Science 12 & Medline 7 & BVS 26 \\
\hline
\end{tabular}

\begin{tabular}{|lll|}
\hline \multicolumn{3}{|c|}{ Publicações pré-selecionadas } \\
PUBMED 27 & LILACS 110 \\
CINAHL 2 & SCOPUS 794 & \\
Web of Science 4 & Medline 3 & BVS 146 \\
\hline
\end{tabular}

\begin{tabular}{|lll|}
\hline \multicolumn{3}{|c|}{ Exclusão segundo os critérios de } \\
elegibilidade \\
PUBMED 20 & LILACS 107 & \\
CINAHL 2 & SCOPUS 775 \\
Web of Science 3 & Medline 2 & BVS 132 \\
\hline
\end{tabular}

\begin{tabular}{|c|c|c|}
\hline \multicolumn{3}{|c|}{ Selecionados para leitura na integra } \\
\hline PUBMED 1 & LILACS 3 & \\
\hline CINAHL 0 & SCOPUS 19 & \\
\hline Web of Scienc & 1 Medline 1 & BVS 14 \\
\hline
\end{tabular}

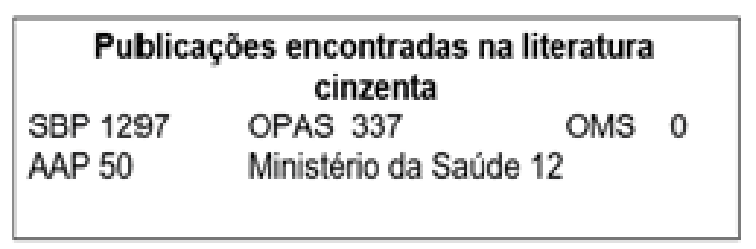

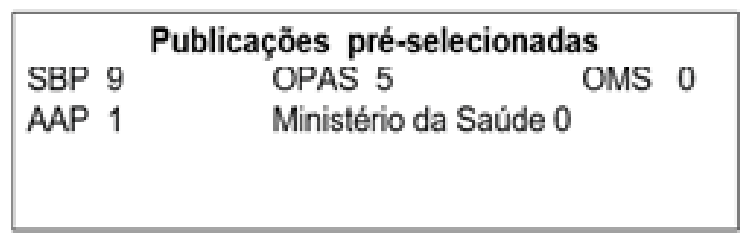

\begin{tabular}{|lll|}
\hline Exclusão segundo os critérios de elegibilidade \\
SBP 9 & OPAS 4 & OMS \\
AAP 1 & Ministério da Saúde 0 & \\
& & \\
\end{tabular}

Selecionados para leitura na integra SBP $0 \quad$ OPAS 1 OMS 0 AAP $0 \quad$ Ministerilo da Saúde 0 
A totalidade dos estudos incluídos nesta revisão foram publicados no ano de 2020. Os países com maior número de publicações foram Espanha $27,29-30,33,35,38$ e China, ${ }^{23-25,37,39-40}$ com seis artigos cada (30\%), seguidos da Turquiaa ${ }^{21,31}$ com duas publicações (10\%) e Austrália, ${ }^{34}$ Itália, ${ }^{22}$ Irã ${ }^{28}$ Índia $^{36}$ e Canadá26 com uma publicação $(5 \%)$ cada. A faixa etária das amostras investigadas variou de três a 23 anos sendo 13 anos a idade média, em uma população total de 1.116 .379 crianças e adolescentes.

Destaca-se que a maior parte (55\%) dos periódicos cujos artigos foram incluídos na presente revisão apresentavam Qualis A e B (Classificação para Área de Enfermagem conforme Plataforma Sucupira de 2012 a $2016 .{ }^{41}$ A classificação por Fator de Impacto variou de 0.746 a 6.936..$^{21-40}$

A apresentação dos estudos incluídos está disposta no Quadro 1:

Quadro 1 - Distribuição dos artigos incluídos na revisão de escopo, conforme população e tipo de estudo, objetivo, 2021. $\mathrm{N}=20$

\begin{tabular}{|c|c|c|c|}
\hline Referência & População & Tipo de estudo & Objetivo \\
\hline 22 & 252 crianças & $\begin{array}{l}\text { Revisão } \\
\text { integrativa }\end{array}$ & $\begin{array}{l}\text { Investigar o impacto da pandemia na saúde mental } \\
\text { das crianças. }\end{array}$ \\
\hline 23 & 2.064 adolescentes & Transversal & Detectar os efeitos psicossociais durante a pandemia. \\
\hline 24 & 7.866 adolescentes & Transversal & $\begin{array}{l}\text { Compreender se há diferença na ansiedade, } \\
\text { depressão e estilo de criação dos pais. }\end{array}$ \\
\hline 25 & 2.050 crianças e adolescentes & Transversal & $\begin{array}{l}\text { Avaliar fatores psicológicos associados ao vício em } \\
\text { Internet durante a pandemia da COVID- } 19 \text {. }\end{array}$ \\
\hline 26 & 3.613 crianças e adolescentes & Transversal & $\begin{array}{l}\text { Demostrar os efeitos psicológicos associados à } \\
\text { pandemia. }\end{array}$ \\
\hline 27 & 1.054 adolescentes & Coorte & $\begin{array}{l}\text { Examinar as relações entre ajuste psicológico e } \\
\text { estresse relatado. }\end{array}$ \\
\hline 28 & 226 adolescentes & Longitudinal & $\begin{array}{l}\text { Estudar as condições de vida e sua associação com } \\
\text { fatores psicológicos. }\end{array}$ \\
\hline 29 & 1512 adolescentes & $\begin{array}{l}\text { Observacional } \\
\text { transversal }\end{array}$ & $\begin{array}{l}\text { Examinar o sofrimento psicológico, vício em internet } \\
\text { e qualidade de vida e insônia. }\end{array}$ \\
\hline 30 & 250 crianças & Qualitativo & $\begin{array}{l}\text { Avaliar como as crianças estão lidando com esta crise } \\
\text { de saúde. }\end{array}$ \\
\hline 31 & 250 crianças & Transversal & $\begin{array}{l}\text { Compreender como representam e enfrentam } \\
\text { emocionalmente a crise de saúde. }\end{array}$ \\
\hline 32 & 745 adolescentes & Transversal & $\begin{array}{l}\text { Determinar os resultados da quarentena e os fatores } \\
\text { psicológicos afetados. }\end{array}$ \\
\hline 33 & 4281 crianças e adolescentes & $\begin{array}{l}\text { Revisão } \\
\text { sistemática }\end{array}$ & $\begin{array}{l}\text { Estabelecer como a solidão e a contenção da doença } \\
\text { impacta na saúde mental. }\end{array}$ \\
\hline 34 & 459 crianças e adolescentes & Transversal & $\begin{array}{l}\text { Abordar os fatores que afetam a saúde mental } \\
\text { durante a pandemia. }\end{array}$ \\
\hline
\end{tabular}


Impactos psicossociais do isolamento social por COVID-19 em crianças, adolescentes... $\mid 8$

\begin{tabular}{|l|l|l|l|}
\hline 35 & 582 adolescentes & Coorte & $\begin{array}{l}\text { Avaliar o impacto do distanciamento físico sobre a } \\
\text { saúde e bem-estar. }\end{array}$ \\
\hline 36 & 1049 crianças & Transversal & $\begin{array}{l}\text { Examinar os efeitos do confinamento sobre as } \\
\text { crianças e suas famílias. }\end{array}$ \\
\hline 37 & 252 crianças e adolescentes & Coorte & $\begin{array}{l}\text { Examinar crianças e adolescentes colocados em } \\
\text { quarentena e impacto psicológico. }\end{array}$ \\
\hline 38 & 4391 crianças e adolescentes. & Transversal & $\begin{array}{l}\text { Estimar o estado de saúde mental durante a } \\
\text { pandemia da Covid-19. }\end{array}$ \\
\hline 40 & 459 crianças e adolescentes & Transversal & $\begin{array}{l}\text { Descrever o impacto psicológico causado pela } \\
\text { pandemia. }\end{array}$ \\
\hline 41 & 2427 crianças e adolescentes & $\begin{array}{l}\text { Longitudinal } \\
\text { experimental }\end{array}$ & $\begin{array}{l}\text { Compreender ostilo de vida de crianças e } \\
\text { adolescentes durantea pandemia da Covid-19. }\end{array}$ \\
\hline
\end{tabular}

As investigações analisadas apontaram os impactos psicossociais do isolamento social pela COVID-19 em crianças e adolescentes, sendo a depressão ${ }^{21,23-30,32-33,35,39-40}$ sinalizada por 14 (70\%) artigos e a ansiedade, ${ }^{21,23-25,27-30,32-33,39-40}$ em seguida, identificada em 12 (60\%) manuscritos.

Os resultados de estudos realizados na China mostraram que o isolamento social, especialmente em locais onde houve lockdown, ocasionou sintomas de ansiedade. ${ }^{25,37,40}$ Crianças e adolescentes chinesas que tiveram parente infectado foram mais propensos a terem sintomas de depressão e inquietação. Aqueles que tinham familiares que trabalhavam com a COVID-19 foram mais sujeitos à prostração. ${ }^{23} \mathrm{~A}$ angústia foi mais frequente em participantes do sexo feminino e residentes em regiões urbanas. ${ }^{25}$ Além disso, a prevalência dos sintomas de tristeza e excitação foi menor para alunos do ensino fundamental e o enfrentamento negativo da pandemia foi identificado como fator de risco para inquietude, melancolia e sintomas de estresse. ${ }^{31}$

Em terceiro lugar, dentre os impactos psicossociais mais frequentes, estão os relatos de medo e preocupação $\mathrm{O}^{21-22,26-27,29-31,36,38,40}$ pelos participantes, apontado em dez artigos (50\%), seguidos de estresse e frustração,,$^{21,24,26-29,31,37,39}$ sendo citados em nove artigos (45\%). Pesquisa evidência que adolescentes se encontravam preocupados e que quanto maior a idade, maior a apreensão. ${ }^{22}$

Pesquisadores realizaram uma coorte no Canadá, com 1.054 adolescentes na faixa etária de 14 a 18 anos, com o intuito de examinar as relações entre ajuste psicológico e estresse relatado 
9 | Santos LC, Pinheiro TJS, Andrade TIX, Sousa PHA, Braga PP, Romano MCC

associado à crise da COVID-19. Os adolescentes mostraram estar preocupados com a crise de COVID-19, particularmente com seus estudos e com seus amigos. O estresse foi associado a maior solidão e aumento da depressão entre os adolescentes que passam mais tempo nas mídias sociais (12\% relatam mais de 10 horas/dia). Essa amostra apresentou baixos níveis de atividade física e altos níveis de estresse. ${ }^{26}$

$\mathrm{Na}$ Espanha, foi realizado um estudo qualitativo com 250 crianças de três a 12 anos, para examinar como elas estão lidando com a crise de saúde devido à COVID-19, durante o isolamento. Foram relatados sentimentos de tristeza, nervosismo, preocupação e solidão. ${ }^{29}$

A dependência digital foi evidenciada por estudos e o aumento do tempo de tela por crianças e adolescentes em isolamento foram descritos em seis (30\%) investigações analisadas. ${ }^{24-26,31,34,39} \mathrm{O}$ aumento do uso da internet durante a pandemia da COVID-19 associou-se com a idade de seis a 18 anos, sexo masculino e sentimentos de depressão e estresse. ${ }^{24}$

As sensações de solidão $(25 \%, \mathrm{n}=5)$, piora do bem-estar emocional $(20 \%, \mathrm{n}=4)$, raiva $(15 \%, \mathrm{n}=3)$ e insônia $(15 \%, \mathrm{n}=3)$ também foram identificados entre crianças e adolescentes participantes dos estudos. $^{21,26-31,34-36,38,40}$ Segundo estudo realizado no Irã, adolescentes que apresentaram sintomas depressivos, ansiedade e estresse estavam mais propensos a desencadearem transtorno relacionado ao jogo e internet, insônia e pior qualidade de vida durante a pandemia da COVID-19.28

Os estudos mostraram que há impactos psicossociais decorrentes do isolamento causado pela COVID-19 em crianças e adolescentes (Quadro 2).

Quadro 2 - Impactos psicossociais do isolamento social em crianças e adolescentes, 2021. N=20

\begin{tabular}{|l|l|}
\hline \multicolumn{1}{|c|}{$\begin{array}{c}\text { Referência } \\
\text { (Autor/ano de publicação) }\end{array}$} & \multicolumn{1}{c|}{ Impactos psicossociais } \\
\hline Aydogdu ALF, $2020^{21}$ & Tristeza, medo, ansiedade, insônia, raiva e estresse \\
\hline Buzzi et al., 202022 & Preocupações e medos \\
\hline Chen; Cheng; Wu; $2020^{23}$ & Depressão e ansiedade \\
\hline Dong H et al; $2020^{24}$ & Depressão, ansiedade, estresse, dependência de internet \\
\hline Duan et al., 202025 & Ansiedade, depressão, dependência de smartphones, dependência de Internet \\
\hline Ellis; Dumas; Forbes; $2020^{26}$ & $\begin{array}{l}\text { Preocupação, estresse elevado, depressão, declínio na atividade física, aumento no uso } \\
\text { da Internet }\end{array}$ \\
\hline Ezpeleta L et al; $2020^{27}$ & Frustração, medo de sair de casa, medo do futuro, insônia \\
\hline
\end{tabular}


Impactos psicossociais do isolamento social por COVID-19 em crianças, adolescentes... $\mid 10$

\begin{tabular}{|l|l|}
\hline Fazeli S et al; $2020^{28}$ & Depressão, insônia, estresse e ansiedade \\
\hline Idoiaga M, et al; $2021^{29}$ & Medo, nervosismo, preocupação, solidão, tristeza, tédio, raiva e sedentarismo \\
\hline Idoiaga M, et al; $2020^{30}$ & Medo, preocupação, tristeza, raiva, opressão, cansaço e até solidão \\
\hline Kilinçel, et al; $2020^{31}$ & Ansiedade e preocupação \\
\hline Loades M Eet al; $2020^{32}$ & Depressão e ansiedade social \\
\hline Massa JLP 2020 & Pior bem-estar psicológico \\
\hline Munasinghe et al., 2020 & $\begin{array}{l}\text { Aumento do uso da Internet, maior tempo de tela, declínio na felicidade e consumo de } \\
\text { fast food }\end{array}$ \\
\hline Romero et al., 2020 & Angústia percebida, problemas emocionais \\
\hline Saurabh K; 2020 & Sofrimento psicológico, preocupação, desamparo e medo \\
\hline Tanga S et al; $2020^{37}$ & Depressão, ansiedade e estresse \\
\hline $\begin{array}{l}\text { Vallejo-Slocker L; } \\
\text { Fresneda; Vallejo; } 2020^{38}\end{array}$ & Preocupações, medo e problemas emocionais \\
\hline Xiang M; Zhang Z, 2020 & Depressão, ansiedade, estresse, angústia, maior tempo de tela \\
\hline Zhang, et al; 2020 & Depressão, ansiedade e estresse \\
\hline
\end{tabular}

\section{Discussão}

A presente revisão observou que o isolamento social, em função da COVID-19, impactou socialmente e psicologicamente crianças e adolescentes. Os estudos revelaram que dentre as repercussões do isolamento na pandemia da COVID-19, para crianças e adolescentes, destacam-se

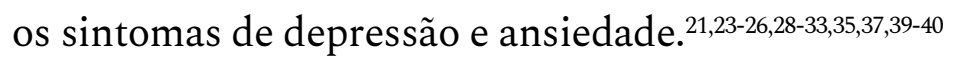

De fato, um monitoramento excessivo da autonomia na infância e na juventude, como é intrínseco ao contexto pandêmico, pode contribuir com sintomas depressivos, o que pode explicar os resultados da presente investigação. ${ }^{42}$ Sintomas depressivos como solidão e tristeza no jovem comprometem o bem-estar psicológico, inibindo os sentimentos e emoções, como alegria, felicidade e boa disposição. Contudo, indivíduos que apresentam maior suporte familiar afetivo têm menores chances de serem acometidas pela depressão. ${ }^{43-44}$ De fato, potencializar, neste momento de crise, relações de afeto entre pais e filhos é essencial para o desenvolvimento e cuidado. ${ }^{45}$

Observou-se também que o estresse compõe o terceiro impacto psicossocial mais prevalente entre crianças e adolescentes durante o isolamento pela Covid-19.21,24,26-29,31,37,39 Pesquisadores mostraram que o confinamento, em casa, de 220 milhões de crianças e adolescentes chineses, incluindo 180 milhões de estudantes de escolas primárias e secundárias e 47 milhões da pré-escola, 
11 | Santos LC, Pinheiro TJS, Andrade TIX, Sousa PHA, Braga PP, Romano MCC

provocará impactos psicológicos. ${ }^{46}$ Todo o contexto negativo da pandemia facilita o aumento dos níveis de estresse da população. As consequências destes estressores relacionados à elevação de casos, o excesso de notícias e o crescente número de óbitos impactam o desenvolvimento e o comportamento da criança, elevando o risco para transtornos mentais agudos e crônicos. ${ }^{47}$

É preciso considerar que atualmente as mídias sociais e seu alcance durante o aparecimento da COVID-19, quando comparada a pandemias anteriores, espalha volume de informações que nem sempre são autênticas e tampouco verificadas, ora criando falsas expectativas de cura, ora provocando medo exacerbado e pânico. Este fenômeno gera insegurança e angústia, que é agravada pela divergência de orientações advindas de autoridades de saúde pública e governamentais. ${ }^{48}$

Diversos problemas psicológicos podem ser decorrentes de uma pandemia. O trauma na primeira infância pode favorecer o risco de uma criança apresentar uma doença mental e da mesma forma regredir o seu desenvolvimento. ${ }^{49}$ Além do mais, experiências adversas na infância podem ocasionar resultados ruins ao longo da vida, como abuso de substâncias e problemas com relacionamentos ou educação, bem como aumentar o risco de doenças crônicas. Muitas crianças em isolamento ou quarentena durante a pandemia de H1N1 alcançaram os critérios para transtorno de estresse pós-traumático (TEPT). ${ }^{49}$

Portanto, faz-se necessária uma maior organização da Atenção à Saúde para assistir à estas demandas da população. Isso significa fazer a busca ativa e acompanhar famílias com risco aumentado para adoecimento, com maiores vulnerabilidades, no sentido de promover educação em saúde para os determinantes de estresse e apoiá-las no processo de luto. Novas formas de assistência também deverão ser planejadas, por exemplo, com o uso de tecnologias e internet. ${ }^{50}$

Medo e preocupação foram identificados nos artigos analisados. ${ }^{21-22,26-27,29-31,36-37,40}$ Preocupação excessiva, irritabilidade, confinamento em casa e medo da infecção e transmissão estão inteiramente ligados a sintomas de inquietação durante a pandemia. A privação social é um fator de risco para o agravamento da saúde mental, incluindo sintomas depressivos, angústia, medo, estresse 
Impactos psicossociais do isolamento social por COVID-19 em crianças, adolescentes... | 12

pós-traumático e insônia. ${ }^{42}$ Com relação aos transtornos mentais, o medo da doença (coronofobia) aumentou os sintomas de angústia, principalmente com o isolamento social. ${ }^{51}$

Outro aspecto refere-se à dependência digital relacionada ao maior tempo de tela. ${ }^{24-26,31,34,39}$ As medidas de contenção da doença impossibilitaram o contato social de crianças e adolescentes o que contribuiu para o aumento do tempo gasto na internet. $\mathrm{O}$ bate papo online e ver as publicações de pessoas próximas trazem benefícios para a saúde mental, incluindo que muitas vezes recebem apoio pela internet dos amigos e familiares em momentos difíceis como este. ${ }^{48}$

Identificou-se que devido ao fechamento das escolas, as atividades de aprendizagem presenciais foram transferidas parcialmente ou totalmente para o ambiente virtual, com trabalhos realizados em casa e aulas digitais, o que adiciona mais tempo de tela ao que já existia antes do confinamento. ${ }^{51-52}$ Em contrapartida, a aprendizagem digital é um sistema restrito que traz à tona as desigualdades socioeconômicas, pois muitas crianças têm acesso limitado, compartilhado ou nenhum acesso online devido à falta de equipamento ou provisão de internet. ${ }^{49}$

Sofrimento psicológico e solidão compõe relatos frequentes de crianças e adolescentes na pandemia. É de conhecimento que 75\% dos adultos que possuem ou já possuíram um problema de saúde mental antes dos 24 anos evidenciaram dificuldade de relacionamento, rejeição, bullying e solidão. Entretanto, colegas que possuem um bom relacionamento diminuem alterações na saúde mental e este favorece a resiliência do adolescente..$^{53}$

O estreitamento de laços por redes sociais, o apoio social de vizinhos, os hábitos de sono e alimentação saudáveis, a música, os exercícios físicos que podem ser feitos em casa e informações precisas da saúde podem contribuir para minimizar os impactos psicossociais do isolamento em crianças e adolescentes. Recomendações de saúde mental durante a pandemia devem envolver a família como um todo, no sentido de manter atividades de lazer dentro dos protocolos estabelecidos, além de limitar notícias relacionadas a pandemia, pois podem acarretar distúrbios de ansiedade. Realizar televisitas por chamadas telefônicas ou de vídeos também são uma opção, além 
do uso de redes sociais que permitam a comunicação à distância, de modo a facilitar a troca de sentimentos neste momento tão confuso. ${ }^{54}$

Outro fator desencadeante de sintomas de estresse é escassez de atividades de lazer no atual contexto e dificuldades de acesso aos serviços de saúde. Os ajustes realizados no sistema de saúde, tanto na atenção básica quanto na especializada, reduziram as oportunidades de assistência, trazendo uma maior vulnerabilidade para esses grupos. Sendo assim, reforça-se a importância de que a atenção à saúde mental e psicossociais estejam acessíveis e apropriadamente adaptados a nova forma de assistência. ${ }^{55}$

Destaca-se que a arte, a cultura e o esporte também desempenham uma função fundamental na consolidação da saúde mental de toda a população, principalmente do público jovem. Campanhas temáticas direcionadas aos jovens representam uma maneira de amenizar os impactos do distanciamento social, táticas para o desenvolvimento da saúde física e mental, monitoramento de sinais de alerta que podem auxiliar as famílias a atravessar essa difícil fase e fortalecer o vínculo com o serviço de saúde. ${ }^{56}$

Ademais, a renovação da assistência por meio da utilização das mídias sociais e teleatendimento, no ambiente da Atenção Primária à Saúde (APS) e Centro de Atenção Psicossocial (CAPS) são novos modos de fazer saúde mental. Cita-se redes sociais como WhatsApp e uso de email para melhorar a interação e atualização do usuário, por meio dos atendimentos coletivos e individualizados. A interação entre centros de referência tem o potencial de favorecer o enfrentamento a diversas eventualidades que colocam crianças, adolescentes e suas famílias em risco pessoal, social e situações de violação de direitos. Assim, novos modos de fazer saúde são essenciais para a reafirmação da autonomia do usuário e o vínculo com o serviço de APS. ${ }^{57}$

Quanto as limitações desta revisão, a ausência de investigações com amostras de crianças e adolescentes brasileiras não permitem generalizações dos resultados, considerando as especificidades culturais, sociais e econômicas deste país. Contudo, a revisão sinaliza para situações 
Impactos psicossociais do isolamento social por COVID-19 em crianças, adolescentes... | 14

de alerta e necessidade de pesquisadores brasileiros se dedicarem a esta temática.

\section{Conclusão}

A presente revisão de escopo mapeou impactos psicossociais ocasionados pelo isolamento pela COVID-19 entre crianças e adolescentes. As repercussões psíquicas têm sido marcadas por quadros de depressão, ansiedade, medo, estresse e insônia o que pode sinalizar para uma piora no bem-estar psicológico desta população. O aumento ou dependência do uso de telas ficou evidente. Precisa-se considerar que, se por um lado a tecnologia pode favorecer encontros virtuais a escassez de interações presenciais poderá limitar o desenvolvimento social do público infantojuvenil. Estudos longitudinais serão relevantes para acompanhar os efeitos, a longo prazo, do isolamento no desenvolvimento social e na saúde mental de crianças e adolescentes.

\section{Referências}

1. Fegert JM, Vitiello B, Plener PL, Clemes V. Challenges and burden of the Coronavirus 2019 (COVID-19) pandemic for child and adolescent mental health: a narrative review to highlight clinical and research needs in the acute phase and the long return to normality. Child Adolesc Psychiatry Ment Health. 2020;14:20. doi: 10.1186/s034-020-00329-3

2. Ministério da Saúde (BR). O que é Covid-19 [Internet]. Brasília, DF: Ministério da Saúde; 2020 [acesso em 2020 nov 25]. Disponível em: https://www.gov.br/saude/pt-br/coronavirus/o-que-e-o-coronavirus

3. Organização Pan-Americana da Saúde (OPAS). Folha informativa COVID-19 [Internet]. Brasília, DF: Organização Pan-Americana da Saúde [acesso em 2021 fev 05]. Disponível em: https://www.paho.org/pt/covid19

4. Ministério da Saúde (BR). Boletim epidemiológico coronavírus - N40. Brasília, DF: Ministério da Saúde; 2020 .

5. Zimmermann P, Curtis N. Coronavirus infections in Children Including COVID-19. Pediatr Infect Dis J. 2020;39(5):355-68. doi: 10.1097/INF.0000000000002660

6. Bi Q, Wu Y, Mei S, Ye C, Zou X, Zhang Z, et al. Epidemiology and transmission of COVID-19 in Shenzhen China: analysis of 391 cases and 1,286 of their close contacts. MedRxiv. 2020. doi: 10.1101/2020.03.03.20028423

7. Riphagen S, Gomez X, Gonzalez-Martinez C, Wilkinson N, Theocharis P. Hyperinflammatory shock in children during COVID-19 pandemic. Lancet. 2020;395(10237):1607-8. doi: 10.1016/S0140-6736(20)31094-1 
8. DeBiasi RL, Song X, Delaney M, Bell M, Smith K, Pershad J, et al. Severe Coronavirus disease-2019 in children and young adults in the Washington, DC, metropolitan region. J Pediatr. 2020;223:199-203. doi: 10.1016/j.jpeds.2020.05.007.

9. Jones VG, Mills M, Suarez D, Hogan CA, Yeh D, Bradley Segal J, et al. COVID-19 and Kawasaki disease: novel virus and novel case. Hosp Pediatr. 2020;10(6):537-40. doi: 10.1542/hpeds.2020-0123

10. Yoshikawa H, Wuermli AJ, Britto PR, Dreyer B, Leckman JF, Lye SJ, et al. Effects of the global Coronavirus disease-2019 pandemic on early childhood development: short- and long-term risks and mitigating program and policy actions. J Pediatr. 2020;223:188-93. doi: 10.1016/j.jpeds.2020.05.020

11. Fundação Oswaldo Cruz (FIOCRUZ). Saúde mental e atenção psicossocial na pandemia Covid-19 [Internet]. Brasília, DF: FIOCRUZ; 2020 [acesso em 2020 maio 25]. Disponível em: https://www.fiocruzbrasilia.fiocruz.br/coronavirus/saude-mental-em-tempos-de-coronavirus/

12. Guan H, Okely AD, Aguilar-Farias N, Del Pozo Cruz B, Draper CE, El Hamdouchi A, et al. Promoting healthy movement behaviours among children during the COVID-19 pandemic. Lancet Child Adolesc Health. 2020;4(6):416-8. doi: 10.1016/S2352-4642(20)30131-0

13. Rodrigues H, Gomes AMM, Oliveira RT, Piccirillo D, Brito RC. Socialização legal de crianças e adolescentes: revisão da literatura e desafios de pesquisa. Plural. 2017;24(1):105-23. doi: 10.11606/issn.21768099.pcso.2017.127330

14. Nobre JNP, Santos JN, Santos LR, Guedes SC, Pereira L, Costa JM, et al. Fatores determinantes no tempo de tela de crianças na primeira infância. Ciênc Saúde Colet. 2021;26(3):1127-36. doi: 10.1590/141381232021263.00602019

15. Fundação Oswaldo Cruz (FIOCRUZ), Instituto Nacional de Saúde da Mulher, da Criança e do Adolescente Fernandes Figueira (IFF). COVID-19 e saúde da criança e do adolescente [Internet]. Rio de Janeiro: IFF/FIOCRUZ; 2020 [acesso em 2021 fev 07]. Disponível em: https://portaldeboaspraticas.iff.fiocruz.br/atencao-crianca/covid-19-saude-crianca-e-adolescente

16. Linhares MBM, Enumo SRF. Reflexões baseadas na Psicologia sobre efeitos da pandemia COVID-19 no desenvolvimento infantil. Estud Psicol (Campinas). 2020;37. doi: 10.1590/1982-0275202037e200089

17. Peters MDJ, Godfrey C, McInerney P, Munn Z, Tricco A, Khalil H. Chapter 11: scoping reviews. In: Aromataris E, Munn Z (editors). Joanna Briggs Institute Reviewer's Manual. Adelaide (AU): The Joanna Briggs Institute; 2020. Cap. 11. doi: 10.46658/JBIMES-20-12

18. Tricco AC, Lillie E, Zarin W, O'Brien K, Colquhoun H, Kastner M, et al. A scoping review on the conduct and reporting of scoping reviews. BMC Med Res Methodol. 2016;16:15. doi: 10.1186/s12874-016-0116-4

19. Romano MCC, Santos LC, Andrade TIX, Pinheiro TJS. psychosocial impacts related to the social isolation dur to Covid-19 in children and adolescents: scoping review [Internet]. Open Science Framework; 2020 [cited 2020 Oct 08]. Available from: https://osf.io/jdgck/

20. Ministério da Mulher, da Família e dos Direitos Humanos (BR), Secretaria Nacional dos Direitos da Criança e do Adolescente. Estatuto da Criança e Adolescente: Lei no 8069, de 13 de julho de 1990 [Internet]. 
Impactos psicossociais do isolamento social por COVID-19 em crianças, adolescentes... | 16

Brasília, DF: Ministério da Mulher, da Família e dos Direitos Humanos; 2019 [acesso em 2020 nov 05]. Disponível em: https://www.gov.br/mdh/pt-br/centrais-de-conteudo/crianca-e-adolescente/estatuto-dacrianca-e-do-adolescente-versao-2019.pdf

21. Aydogdu ALF. Saúde mental das crianças durante a pandemia causada pelo novo coronavírus: revisão integrativa. J Health NPEPS [Internet]. 2020 [acesso em 2021 mar 10];5(2):e4891. Disponível em: https://periodicos.unemat.br/index.php/jhnpeps/article/view/4891

22. Buzzi C, Tucci M, Ciprandi R, Brambilla I, Caimmi S, Ciprandi G, et al. The psycho-social effects of COVID-19 on Italian adolescents' attitudes and behaviors. Ital J Pediatr. 2020;46(1):69. doi: 10.1186/s13052020-00833-4

23. Chen S, Cheng Z, Wu J. Risk factors for adolescents' mental health during the COVID-19 pandemic: a comparison between Wuhan and other urban areas in China. Global Health. 2020; 16(1):96. doi: 10.1186/s12992-020-00627-7

24. Dong H, Yang F, Lu X, Hao W. Internet addiction and related psychological factors among children and adolescents in China during the coronavirus disease 2019 (COVID-19) epidemic. Front Psychiatry. 2020;11:00751. doi: 10.3389/fpsyt.2020.00751

25. Duan L, Shao X, Wang Y, Huang Y, Miao J, Yang X, et al. An investigation of mental health status of children and adolescents in china during the outbreak of COVID-19. J Affect Disord. 2020; 275:112-8. doi: 10.1016/j.jad.2020.06.029

26. Ellis WE, Dumas TM, Forbes LM. Physically isolated but socially connected: psychological adjustment and stress among adolescents during the initial COVID-19 crisis. Can J Behav Sci 2020;52(3):177-87. doi: $10.1037 / \mathrm{cbs} 0000215$

27. Ezpeleta L, Navarro JB, De la Osa N, Trepat E, Penelo E. Life conditions during COVID-19 lockdown and mental health in Spanish adolescents. Int J Environ Res Public Health 2020;17(19):7327. doi: 10.3390/ijerph17197327

28. Fazeli S, Mohammadi Zeidi I, Lin CY, Namdar P, Griffiths MD, Ahorsu DK, et al. Depression, anxiety, and stress mediate the associations between internet gaming disorder, insomnia, and quality of life during the COVID-19 outbreak. Addict Behav Rep. 2020;12:100307. doi: 10.1016/j.abrep.2020.100307

29. Idoiaga Mondragon N, Berasategi Sancho N, Dosil Santamaria M, Eiguren Munitis A. Struggling to breathe: a qualitative study of children's wellbeing during lockdown in Spain. Psychol Health. 2021;36(2):17994. doi: 10.1080/08870446.2020.1804570

30. Idoiaga Mondragon N, Berasategi Sancho N, Eiguren Munitis A, Picaza M. Exploring children's social and emotional representations of the COVID-19 pandemic. Front Psychol. 2020;11:1952. doi: 10.3389/fpsyg.2020.01952

31. Kılınçel Ş, Kılınçel O, Muratdağ in home-quarantine during COVID-19 pandemic in Turkey. Asia Pac Psychiatry. 2021;13(2):e12406. doi: 10.1111/appy.12406 
32. Loades ME, Chatburn E, Higson-Sweeney N, Reynolds S, Shafran R, Brigden A, et al. Rapid systematic review: the impact of social isolation and loneliness on the mental health of children and adolescents in the context of COVID-19. J Am Acad Child Adolesc Psychiatry. 2020;59(11):1218-39e3. doi: 10.1016/j.jaac.2020.05.009

33. Pedreira Massa JL. Salud mental y COVID-19 en infancia y adolescencia: visión desde la psicopatología y la salud pública. Rev Esp Salud Pública [Internet]. 2020 [acceso 2021 ene 20];94:e1-17. Disponible en: https://www.mscbs.gob.es/biblioPublic/publicaciones/recursos_propios/resp/revista_cdrom/VOL94/C_ESPEC IALES/RS94C_202010141.pdf

34. Munasinghe S, Sperandei S, Freebairn L, Conroy E, Jani H, Marjanovic S, et al. The impact of physical distancing policies during the covid-19 pandemic on health and well-being among australian adolescents. J Adolesc Health. 2020;67(5):653-61. doi: 10.1016/j.jadohealth.2020.08.008

35. Romero E, López-Romero L, Domínguez-Álvarez B, Villar P, Gómez-Fraguela JA. Testing the effects of COVID-19 confinement in Spanish children: the role of parents' distress, emotional problems and specific parenting. Int J Environ Res Public Health. 2020;17(19):6975. doi: 10.3390/ijerph17196975

36. Saurabh K, Ranjan S. Compliance and psychological impact of quarantine in children and adolescents due to Covid-19 pandemic. Indian J Pediatr. 2020;87(7):532-6. doi: 10.1007/s12098-020-03347-3

37. Tang S, Xiang M, Cheung T, Xiang YT. Mental health and its correlates among children and adolescents during COVID-19 school closure: The importance of parent-child discussion. J Affect Disord. 2021;279:35360. doi: $10.1016 /$ j.jad.2020.10.016.

38. Vallejo-Slocker L, Fresneda J, Vallejo MA. Psychological wellbeing of vulnerable children during the COVID-19 pandemic. Psicothema. 2020;32(4):501-7. doi: 10.7334/psicothema2020.218

39. Xiang M, Zhang Z, Kuwahara K. Impact of COVID-19 pandemic on children and adolescents' lifestyle behavior larger than expected. Prog Cardiovasc Dis. 2020;63(4):531-2. doi: 10.1016/j.pcad.2020.04.013

40. Zhang C, Ye M, Fu Y, Yang M, Luo F, Yuan J, et al. The psychological impact of the COVID-19 pandemic on teenagers in China. J Adolesc Health 2020;67(6):747-55. doi: 10.1016/j.jadohealth.2020.08.026

41. Coordenação de Aperfeiçoamento de Pessoal de Nivel Superior (CAPES). Plataforma Sucupira. Qualis classificações de periódicos [Internet]. 2021 [acesso em 2021 abril 01]. Disponível em: https://sucupira.capes.gov.br/sucupira/public/consultas/coleta/veiculoPublicacaoQualis/listaConsultaGeralPeriodicos.jsf 42. Yap MBH, Pilkington PD, Ryan SM, Jorm AF. Parental factors associated with depression and anxiety in young people: a systematic review and meta-analysis. J Affect Disord. 2014;156:8-23. doi: 10.1016/j.jad.2013.11.007

43. Coutinho MPL, Pinto AVL, Cavalcanti JG, Araújo LS, Coutinho ML. Relação entre depressão e qualidade de vida de adolescentes no contexto escolar. Psicol Saúde Doenças. 2016;17(3):338-51. doi: $10.15309 / 16$ psd170303

44. Miliauskas CR, Faus DP. Saúde mental de adolescentes em tempos de Covid-19: desafios e possibilidades de enfrentamento. Physis. 2020;30(4):e300402. doi: 1590/s0103-73312020300402 
Impactos psicossociais do isolamento social por COVID-19 em crianças, adolescentes... | 18

45. Veronese JRP, Machado CAA, Pozzoli L. Pandemia, direito e fraternidade: um mundo novo nascerá [Internet]. Caruaru, PE: Asces-Unita; 2020 [acesso em 2020 nov 05]. Disponível em: http://repositorio.asces.edu.br/handle/123456789/2632

46. Wang G, Zhang Y, Zhao J, Zhang J, Jiang F. Mitigate the effects of home confinement on children during the COVID-19 outbreak. Lancet. 2020;395(10228):945-7. doi: 10.1016/S0140-6736(20)30547-X

47. Mangueira LFB, Negreiros RAM, Diniz MFFM, Sousa JK. Saúde mental das crianças e adolescentes em tempos de pandemia: uma revisão narrativa. Rev Eletrônica Acervo Saúde. 2020; 12(11):e4919. doi:10.25248/reas.e4919.2020

48. Primo A. Afetividade e relacionamentos em tempos de isolamento social: intensificação do uso de mídias sociais para interação durante a pandemia de COVID-19. Comun Inov. 2020;21(47):176-98. doi:10.13037/ci.vol21n47.7283

49. Ashikkali L, Carroll W, Johnson C. The indirect impact of COVID-19 on child health. Paediatr Child Health (Oxford). 2020;30(12):430-7. doi: 10.1016/j.paed.2020.09.004

50. Nabuco G, Oliveira MHPP, Afonso MPD. O impacto da pandemia pela COVID-19 na saúde mental: qual é o papel da Atenção Primária à Saúde? Rev Bras Med Fam Comunidade. 2020;15(42):2532. doi: $10.5712 / \mathrm{rbmfc} 15(42) 2532$

51. Stavridou A, Stergiopoulou AA, Panagouli E, Mesiris G, Thirios A, Mougiakos T, et al. Psychosocial consequences of COVID-19 in children, adolescents and young adults: a systematic review. Psychiatry Clin Neurosci. 2020;74(11):615-6. doi: 10.1111/pcn.13134

52. López-Bueno R, López-Sánchez GF, Casajús JA, Calatayud J, Gil-Salmerón A, Grabovac I, et al. Healthrelated behaviors among school-aged children and adolescents during the Spanish Covid-19 confinement. Front Pediatr. 2020;8:573. doi: 10.3389/fped.2020.00573

53. Orben A, Tomova L, Blakemore SJ. The effects of social deprivation on adolescent development and mental health. Lancet Child Adolesc Health. 2020;4(8):634-40. doi: 10.1016/S2352-4642(20)30186-3

54. Bungi SC, Pires RS, Bazzan EM. Revisitando as relações familiares nos tempos de covid-19. Rev GETS [Internet]. 2020 [acesso $2021 \quad$ abr 06];3(2):101-15. Disponível em: https://ojs3x.gets.science/index.php/gets/article/view/75

55. Fundo das Nações Unidas para a Infância (UNICEF). Orientação para a prevenção e o controle da COVID-19 nas escolas. Conteúdo suplementar C: saúde mental e apoio psicossocial [Internet]. Brasília, DF: UNICEF; 2020 [acesso em 2021 abr 06]. Disponível em: https://www.unicef.org/lac/media/12331/file

56. Király O, Potenza MN, Stein DJ, King DL, Hodgins DC, Saunders JB, et al. Preventing problematic Internet use during the COVID-19 pandemic: consensus guidance. Compr Psychiatry. 2020;100:152180. doi: 10.1016/j.comppsych.2020.152180.

57. Cruz NMLV, Souza EB, Sampaio CSF, Santos AJM, Chaves SV, Hora RN, et al. Apoio psicossocial em tempos de COVID-19: experiências de novas estratégias de gestão e ajuda mútua no sul da Bahia, Brasil. Aps Rev. 2020;2(2):97-105. doi: 10.14295/aps.v2i2.94 
19 | Santos LC, Pinheiro TJS, Andrade TIX, Sousa PHA, Braga PP, Romano MCC

Editora Científica: Cristiane Cardoso de Paula

Editora Associada: Aline Cammarano Ribeiro

\section{Autor correspondente}

Márcia Christina Caetano Romano

E-mail: marciachristinacs@ufsj.edu.br

Endereço: Rua Belvedere, 530, Manoel Valinhas, Divinópolis-MG CEP: 35500-287

\section{Contribuições de Autoria}

\section{1 - Letícia Camilo Santos}

Concepção ou desenho do estudo/pesquisa, análise e/ou interpretação dos dados.

\section{2 -Tayná July Silva Pinheiro}

Concepção ou desenho do estudo/pesquisa, análise e/ou interpretação dos dados.

\section{3 - Thayane Ingrid Xavier de Andrade}

Concepção ou desenho do estudo/pesquisa, análise e/ou interpretação dos dados.

\section{4 - Márcia Christina Caetano Romano}

Concepção ou desenho do estudo/pesquisa, análise e/ou interpretação dos dados, revisão final com participação crítica e intelectual no manuscrito.

\section{5 - Paulo Henrique Alves Sousa}

Concepção ou desenho do estudo/pesquisa, análise e/ou interpretação dos dados.

\section{6 - Patrícia Pinto Braga}

Concepção ou desenho do estudo/pesquisa, revisão final com participação crítica e intelectual no manuscrito.

\section{Como citar este artigo}

Santos LC, Pinheiro TJS, Andrade TIX, Sousa PHA, Braga PP, Romano MCC. Psychosocial impacts of social isolation due to COVID-19 in children, adolescents and young people: a scoping review. Rev. Enferm. UFSM. 2021 [Accessed on: Year Month Day]; vol.11 e73: 1-19. DOI: https://doi.org/10.5902/2179769265407 